\title{
Access Control Application using Android Smartphone, Arduino and Bluetooth
}

\author{
Salonie Vyas \\ B.E. Computer \\ Engineering student, \\ Sinhgad Academy of \\ Engineering, Pune, \\ Maharashtra, India
}

\author{
Umang \\ Chaudhari \\ B.E. Computer \\ Engineering student, \\ Sinhgad Academy of \\ Engineering, Pune, \\ Maharashtra, India
}

\author{
V. Chinmay \\ Nandini \\ B.E. Computer \\ Engineering student, \\ Sinhgad Academy of \\ Engineering, Pune, \\ Maharashtra, India
}

\author{
Bhushan Thakare \\ Asst. Professor, \\ Sinhgad Academy of \\ Engineering, Pune, \\ Maharashtra, India
}

\begin{abstract}
The Digital India Movement has spawned the need for developing modern techniques and ways to approach and deal with existing problems so that they can be dealt with efficiently and in less time. The idea is not just limited to the design of smart things and software but also to the thinking and approach used by the developers. This may include approaches like facilitating the customer so that he/she has lesser problems to deal with and little to no hurdle in using the particular technology.

In the backdrop of this movement the smartphone has emerged as the major contributor and plays a major role in providing the user with all sorts of interfaces with the surrounding technology. The biggest convenience for the user is to deploy services that take advantage of all the existing features that come built-in with the smart phone. Some of these features are always available with all smart phones are Bluetooth and Wi-fi. One of the important aspects worth looking into is controlling access, maintaining and controlling the flow of traffic and possibly improving the toll collection by upgrading from manual to automatic using internet connected server databases.
\end{abstract}

\section{General Terms}

Bluetooth, Smart City, Wireless Access Control, Automation, Smart phones, Arduino Uno embedded development board, SQL database, PHP, Android.

\section{Keywords}

Android, Bluetooth, Smart phone, Smart city, Arduino Uno, Access Control.

\section{INTRODUCTION}

Due to the convergence of information and communication technologies, smart cities are quickly becoming a reality ${ }^{[1]}$. The wide use of mobile devices makes it the natural target for development in smart cities especially for those social groups that make less use of public e-services. The mobile has managed to reach out to such groups of people. Thus existing e-services can be adapted or new ones can be developed to target broad range of citizens. Also in the Digital India movement mobile is one of the areas whose penetration directly affects GDP, thus it has been labeled as significantly important in the Digital India movement ${ }^{[2]}$.

Android the open source Google mobile OS has a major smart phone market share, attracting wide development opportunities to integrate it in embedded and mobile applications at the smart city level like the location aware mobile services ${ }^{[3]}$.Some of the in-built features that come in all smart phones in Bluetooth, the wireless communication standard that enables data exchange between devices connected by Bluetooth. Bluetooth applications built on Android are gaining popularity in fields of automation and research $^{[4]}$

For projects that require a second remote/ intermediary device the Arduino Uno has been very popular. It is an open-source hardware and computing platform, an embedded microcontroller board. The ability of the Arduino Uno to interact with sensors and communicate with the computer/ mobile devices offers significant advantages for enthusiasts and students to develop interactive applications ${ }^{[5]}$.

\section{BLUETOOTH BASED ACCESS CONTROL}

The problem area worked upon is access control, i.e. the identification and allowance or prohibition of the entry of users in certain locations like toll plazas or societies, etc. In contrast to the existing system in use the proposed system is that which uses an Android smart phone with Bluetooth functionality running a client side App for access control. Certain changes to the access control mechanism are also proposed. These changes include the use of Arduino Uno for interfacing the mechanism to provide/ prohibit access using Bluetooth communication. Data collection in the form of logs stored in an SQL database is provided. The major parts of the access control solution are as follows:

\subsection{Android App}

Since Android is used in a number of development projects many of which have comfortable proximity with the concept of smart cities, it was the platform of choice for user end device ${ }^{[5]}$. A companion application developed in Android Studio running at the user side will be installed on the user's smart phone. This app will take care of the sign-up, login, authentication, log generation and Bluetooth communication with the access control mechanism.

The development included APIs to access the web for retrieval/ storage of data in a web based database (explained in 2.4). Thus Wi-fi connectivity is required for the app to work and Bluetooth functionality is exploited so that the user does not have to care about the underlying complexities. Broadcast receivers take care of events from turning on Bluetooth to discovery of devices to establishing a connection with the Arduino Uno all without user intervention. Options are provided for storing the login credentials for automatic sign-in as well. Following figures show flow of control within the application 


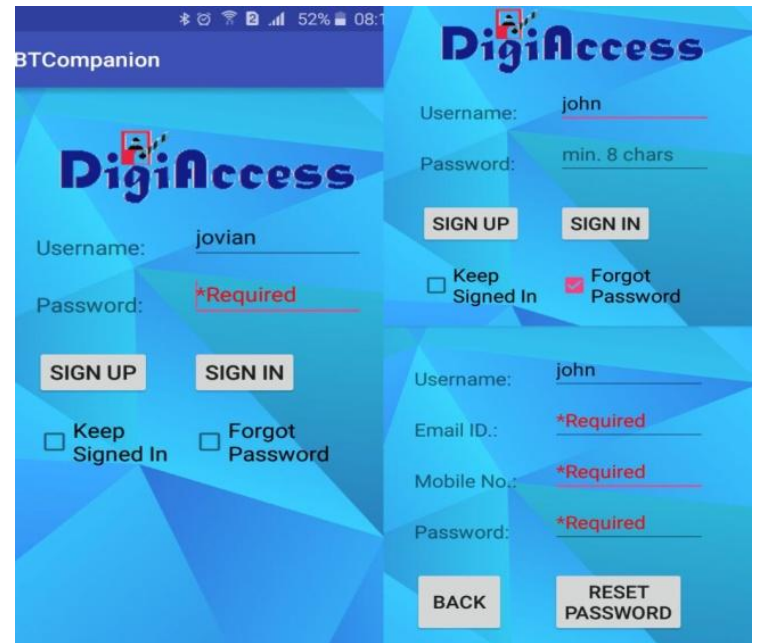

Figure 1: Sign IN/Forgot Password Screen

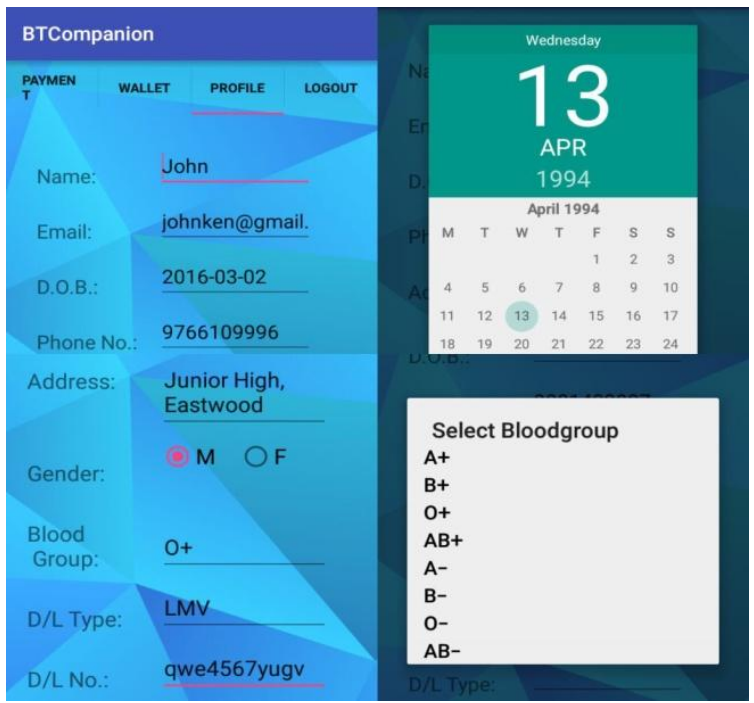

Figure 2: Sign Up Screen

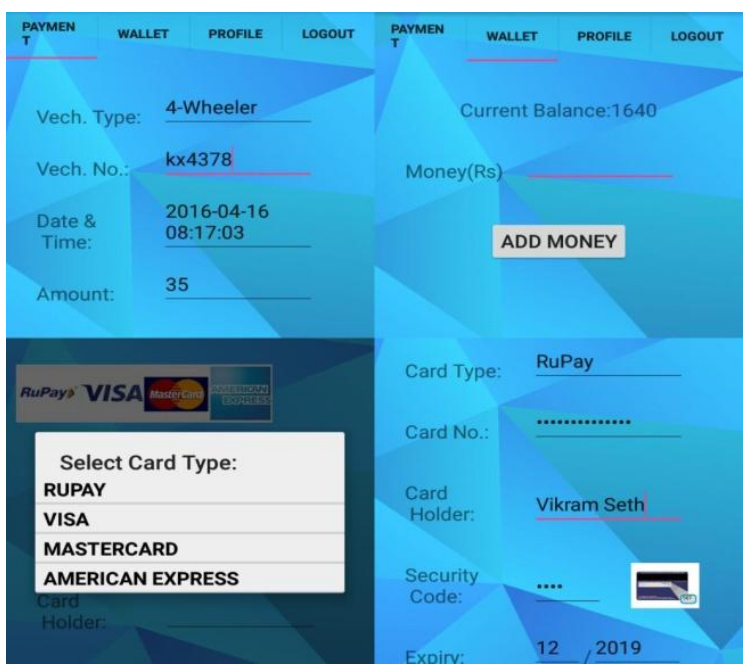

Figure 3: Transaction Screen

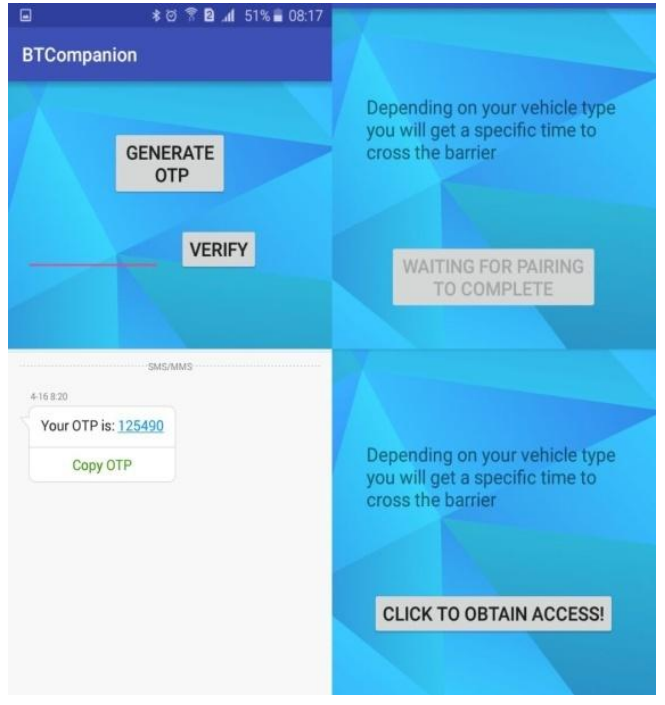

Figure 4: Access Control Screen

\subsection{Arduino}

The Arduino Uno works at the other end of the user, i.e. at the access control side to receive the user's access state (approved or unapproved). The small embedded development board interfaces with a Bluetooth module and a motor IC to drive a motor that will relay the barrier/ gate for access control. Being open-source in nature similar projects have been developed before for Bluetooth interfacing ${ }^{[6]}$, thus it was easy to take advantage of the open-source nature of the board, for a dry test proof-of-concept run. The code base was later heavily customized for suiting the purpose of connectivity required by the access control project. A special Arduino IDE is used for programming the Arduino by uploading sketches, which are Java/ C++ programs that are compiled to produce binary machine code for the Arduino. ${ }^{[7]}$ The sketch uploaded in case of the access control takes care of the communications part between the Android smart phone application.

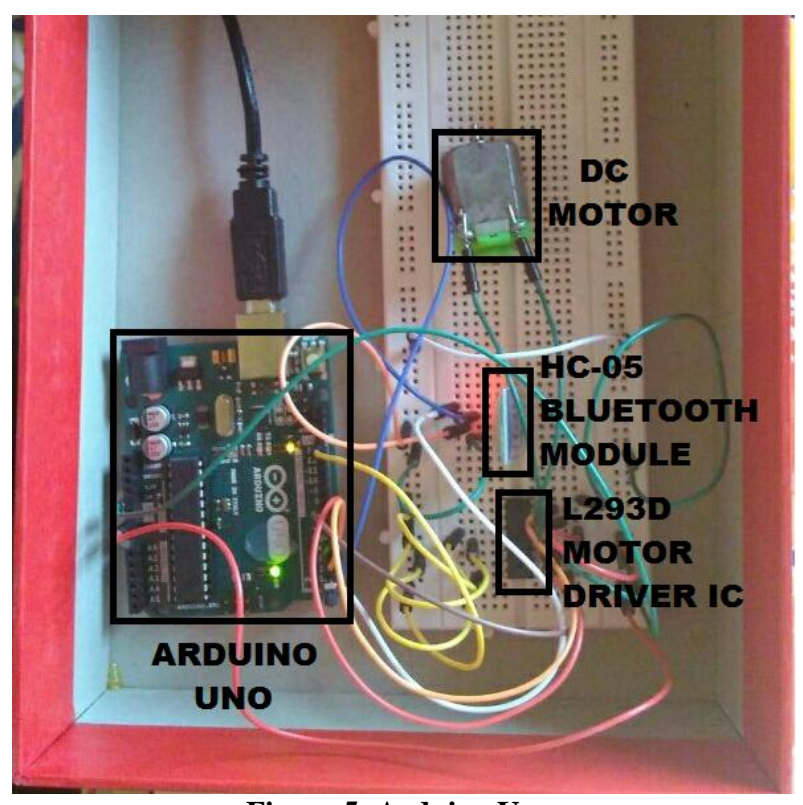

Figure 5: Arduino Uno 


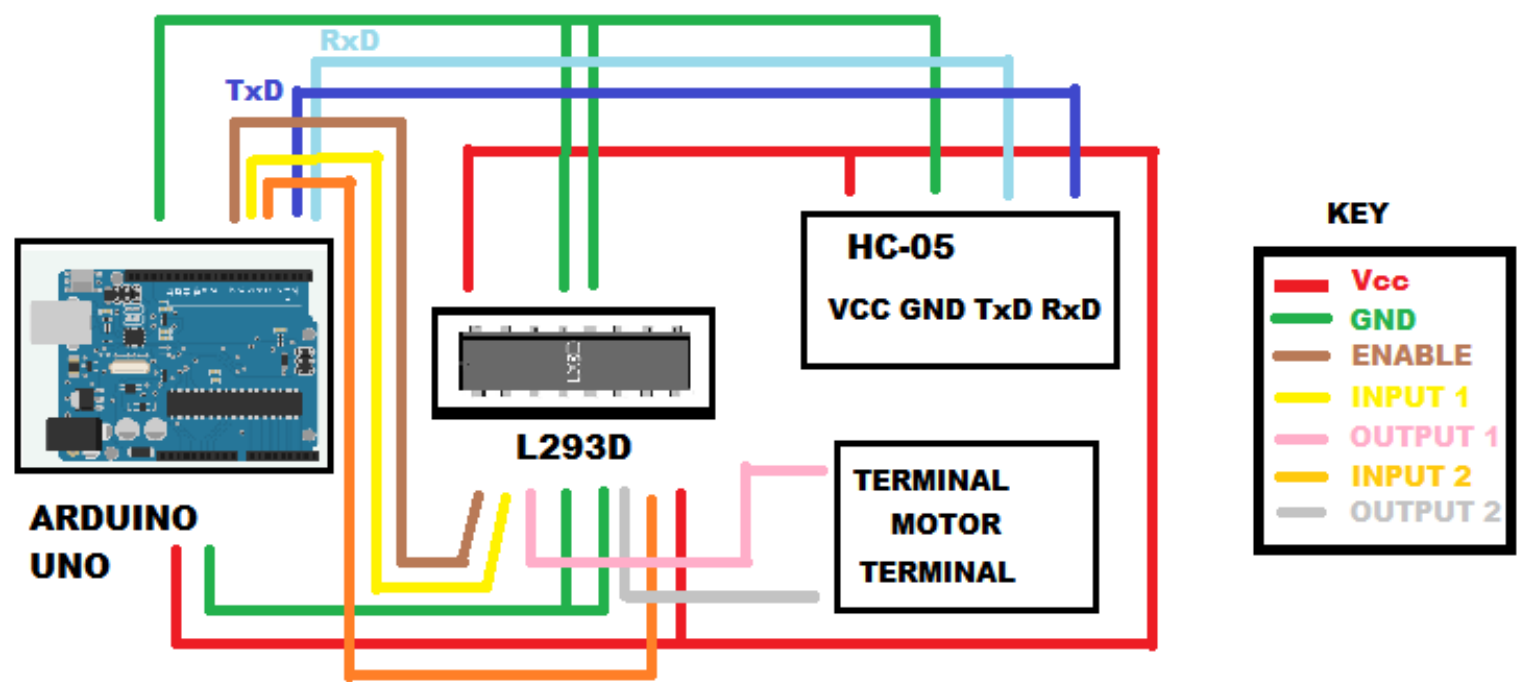

Figure 6: Interface Diagram

\subsection{Bridge}

The Arduino Uno does not have built-in Bluetooth capability but has an interface to send and receive RS-232 compatible signals, thus requiring hardware in form of a Bluetooth transceiver module, the HC-05. Bluetooth Technology is a wireless technology for exchanging information over a typically short range. It is the bridging technology between the Arduino Uno and the Android smart phone used in the access control. Bluetooth has a very important role to play in the development of smart cities and is an emerging platform for future telecommunications and thus has a lot to offer. The second interface was between the Arduino and the motor to drive the access control, the L293d motor driver IC; it takes signals from the Arduino Uno and can alternate the voltage levels to give a bi-directional rotation for the motor. All that is needed is interfacing of gears to drive a barrier or a sliding gate, whichever the case for access control ${ }^{[8]}$.

\subsection{SQL Database}

A website with SQL database connectivity was used for the specific purpose of data management. As mentioned in section 2.1, PHP scripts were used for APIs used in the Android app. A call to the PHP URL and by passing parameters the job of data storage and retrieval was taken care of.

\begin{tabular}{|c|c|c|c|c|c|c|}
\hline Id & Username & Veh_type & Veh_no & Date & Time & Amt_paid \\
\hline 104 & john & 3-Wheeler & $k \times 4254$ & $2016-03-27$ & $05: 32: 19$ & 25 \\
\hline 105 & john & 2-Wheeler & kh7896 & $2016-03-27$ & 05:38:00 & 15 \\
\hline 106 & john & 2-Wheeler & jg5678 & $2016-03-27$ & $15: 17: 50$ & 15 \\
\hline 107 & john & 2-Wheeler & jh2455 & 2016-03-27 & $15: 21: 31$ & 15 \\
\hline
\end{tabular}

Figure 7: Log Table in Database

For the bulk of data at places where required, JSON was also used. Structured data, primarily relational based is used for record storage. The records contains data about the login credentials, personal information, logs of vehicle passage and some pseudo wallet based credentials (more about the pseudo wallet in section 7).

\begin{tabular}{|l|l|} 
Username & Password \\
\hline so & b807023f87e63b8ada92f79f546ff9cc \\
\hline qwer & 962012d09b8170d912f0669f6d7d9d07 \\
\hline mky & 818d1453e5a358243fe3907fc0522776 \\
\hline john & f632fa6f8c3d5f551c5df867588381ab \\
\hline
\end{tabular}

Figure 8: Password stored as a hash

\section{DEVELOPMENT}

For developing a prototype and proof of concept work MIT App Inventor was used. MIT Appinventor is an open source web based application used for Android Application development. It was conceived by Google and later given to Massachusetts Institute of Technology for its further development and maintenance ${ }^{[9]}$. Initial development centered on the communication with Bluetooth and Arduino. Later development was dedicated to data storage and record management. Once the prototype was up and running the main task of building the Android app began. For the SQL Database, web hosting from GoDaddy was purchased, simply because it offered service support, web hosting control panel and database support. 


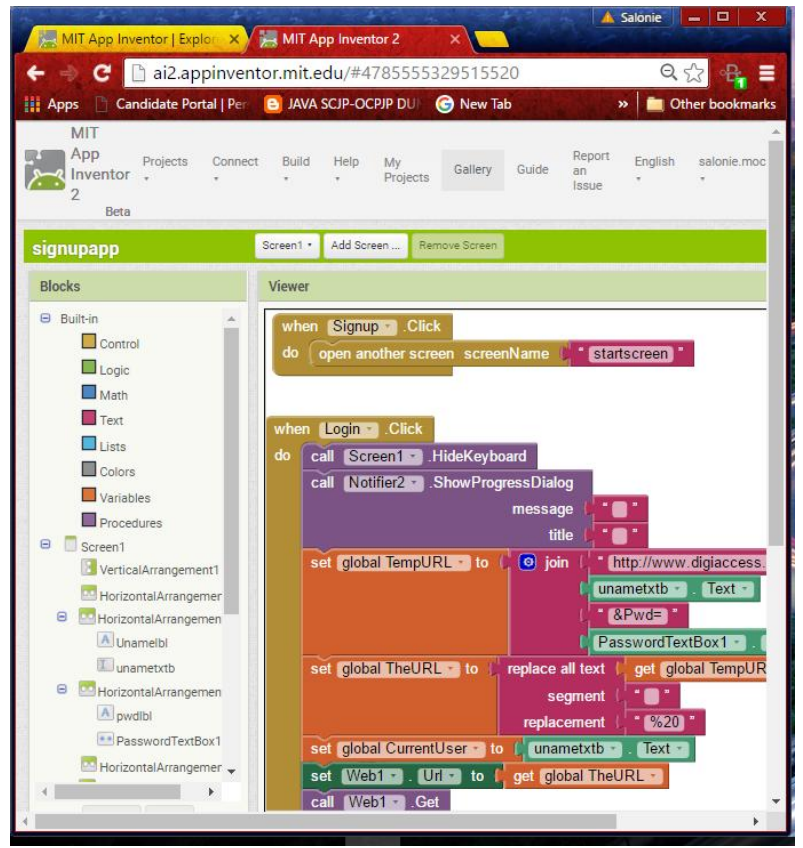

Figure 9: MIT Appinventor Blocks

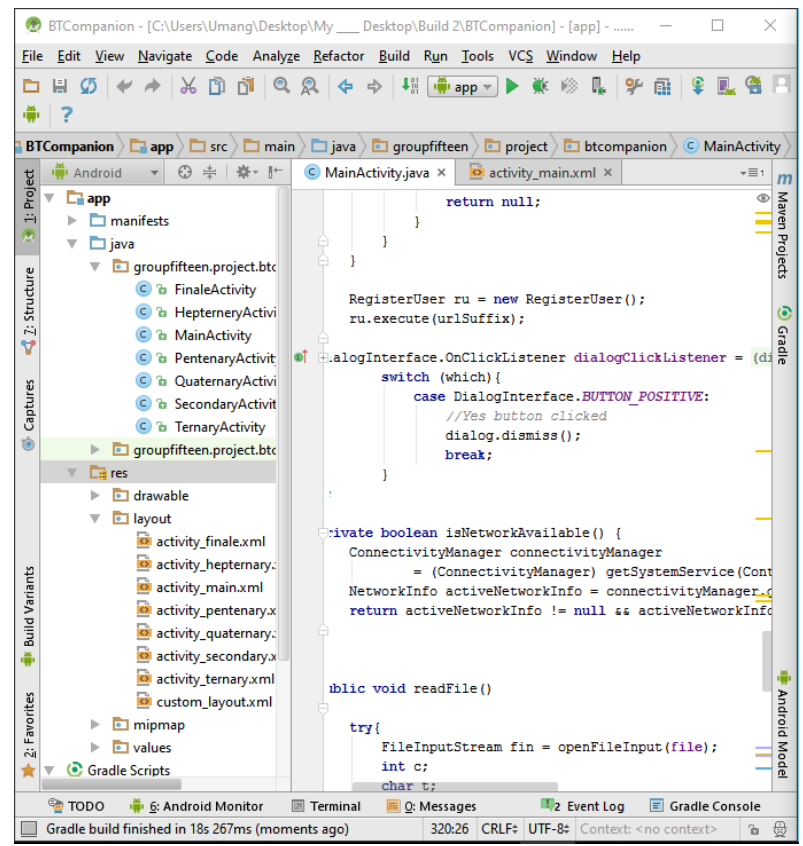

Figure 10: Android Studio IDE

Android Studio IDE was used for Android app development, primarily because it is the recommended platform to develop Android applications on and is also very easy and convenient to use. The GUI design is easy to make by providing a layout editor with drag and drop functionality. The built-in tools make it easy to spot possible error conditions and provide fixes. The Arduino development was done using an Arduino IDE, a cross platform application for uploading sketches to the Arduino ${ }^{[7]}$.

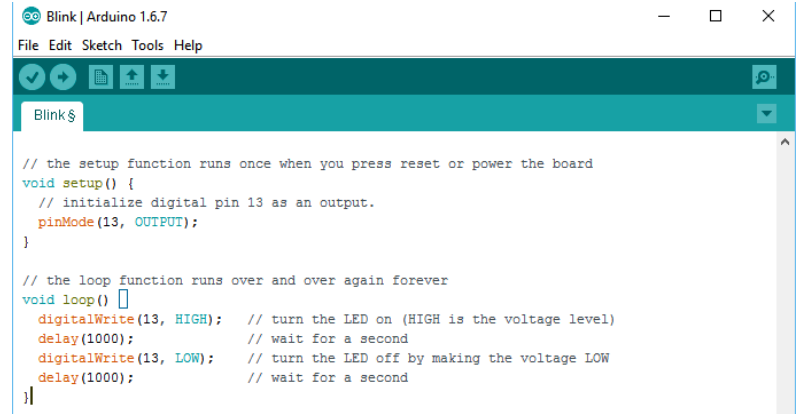

Figure 11: Arduino IDE

\section{RESULTS}

The working scenario of the access control application is such, a user has an Android smart phone with Bluetooth and Internet connectivity and the access control application included. The appropriate setup of the access control hardware is done at the site of restricting access (toll plaza, society, mall parking), with the described Bluetooth transceiver, Arduino motor and interfacing bridge (as described in section 2.3). The user creates an account by signing up, then logs in for each successive use, selects the vehicle type and number, initiates the transaction that ends with the OTP verification. A successful transaction concludes the pairing and communication of access provision between the smart phone and the access control device. A pseudo wallet has been added for managing amount balance in the application. The Bluetooth pairing and communication phase is completely autonomous and not dependent on the user input.

\section{BENEFITS}

- Automated Wireless access control provided.

- No separate device required, works on existing smart phone with user.

- Economically Feasible to implement worldwide including developing countries.

- Manual Labor Reduced

- Can be easily integrated with existing wallet applications.

\section{CHALlengeS}

- Equipment required to be installed at every access control.

- Dependent on user side infrastructure i.e. Android smart phone assuming every user has one.

- Possible interference / conflicts in the frequency range.

- Project at post-ideation stage, no mass scale implementation yet, thus requirements can vary, and development changes may be required.

\section{FUTURE SCOPE}

The design in the current version of the access control is somewhat rigid, but the future editions could hold flexible information like separate vehicle information regarding multiple vehicles of the user. One of the screens designed for the Android app namely the payment screen is a pseudo wallet, i.e. it does not hold actual money, but the API can be extended for managing actual wallet applications. The PHP scripts use traditional name handling techniques but can be enhanced with property files for quick table reordering and 
changes in structure that will not affect the PHP files and remove the need for reflecting the changes in PHP files. The missing administrative section on the website can be added for troubleshooting at the access control. The use case: should a user encounter problems in the app and void access, the administrator would be able to intervene and help with the issue, resolve it and avoid any inconvenience. The concept of access control is flexible and designed to be used not only for toll plazas but also in community parking sites like malls, or societies where it is increasingly becoming a nuisance to manage entry of outsiders. A simple adaptation will potentially solve the major hassle.

\section{CONCLUSION}

In the backdrop of smart cities the access control problem can be given an automated solution. By bringing the solution scope to the user in form of Android app the burden on user is relieved. The user simply has to download an app to obtain access, such ease in solution is expected in the scope of smart cities, but technology also plays a major part. Here the solution is primarily Bluetooth. But other technologies can play an interesting role to evolve and develop the existing system.

\section{REFERENCES}

[1] Smart Cities of the Future by Michael Batty, Kay Axhausen, Giannotti Fosca, Alexei Pozdnoukhov, Armando Bazzani, Monica Wachowicz, Georgios Ouzounis, and Yuval Portugali, UCL working paper series, paper 188-Oct 12, retrieved 3 March 2016

[2] Digital India: Unleashing Prosperity, Confederation of Indian Industry, White paper
[3] Location-aware Mobile Services for a Smart City: Design, Implementation and Deployment by Luca Calderoni, Dario Maio and Paolo Palmieri, Journal of Theoretical and Applied Electronic Commerce Research, retrieved 20 February 2016

[4] Android Application Based Real Time Home Automation by Kallakunta. Ravi Kumar and Shaik Akbar, Indian Journal Of Applied Research, retrieved 15 February 2016

[5] Smart Home Automated Control System Using Android Application and Microcontroller by Mohamed Abd ElLatif Mowad, Ahmed Fathy, Ahmed Hafez, International Journal of Scientific \& Engineering Research, Volume 5, Issue 5, May-2014

[6] Android Mobile Phone Controlled Bluetooth Robot Using 8051 Microcontroller by Ritika Pahuja, Narender Kumar, International Journal of Scientific Engineering and Research, retrieved 2 April 2016

[7] "Programming Arduino Getting Started with Sketches", McGraw-Hill, Nov 8, 2011. Retrieved 2013-03-28.

[8] Three way controlled android Smartphone based robotic vehicle via Bluetooth by Arita Dey, Akash Pal, Sayantan Nandi, Lusika Roy, International Journal of Advanced Research in Computer and Communication Engineering, Vol. 4, Issue 9, September 2015

[9] Hardesty, Larry (August 19, 2010), "The MIT roots of Google's new software", MIT News Office, http://news.mit.edu/2010/android-abelson-0819 